3-13-2020

\title{
Sleeping time is associated with functional limitations in a national sample of older Americans
}

\author{
Brenda M. Vincent \\ Nathaniel Johnson \\ Grant R. Tomkinson \\ University of North Dakota, grant.tomkinson@und.edu \\ Ryan McGrath \\ Brian C. Clark
}

See next page for additional authors

How does access to this work benefit you? Let us know!

Follow this and additional works at: https://commons.und.edu/ehb-fac

Part of the Epidemiology Commons, and the Geriatrics Commons

\section{Recommended Citation}

Brenda M. Vincent, Nathaniel Johnson, Grant R. Tomkinson, et al.. "Sleeping time is associated with functional limitations in a national sample of older Americans" (2020). Education, Health \& Behavior Studies Faculty Publications. 51.

https://commons.und.edu/ehb-fac/51

This Article is brought to you for free and open access by the Department of Education, Health \& Behavior Studies at UND Scholarly Commons. It has been accepted for inclusion in Education, Health \& Behavior Studies Faculty Publications by an authorized administrator of UND Scholarly Commons. For more information, please contact und.commons@library.und.edu. 


\section{Authors}

Brenda M. Vincent, Nathaniel Johnson, Grant R. Tomkinson, Ryan McGrath, Brian C. Clark, and Bong-Jin Choi 


\section{Sleeping Time is Associated with Functional Limitations in a National Sample of Older}

\section{Americans}

Brenda M. Vincent, ${ }^{1}$ Nathaniel Johnson, ${ }^{2}$ Grant R. Tomkinson, ${ }^{3,4}$ Ryan McGrath, ${ }^{2}$ Brian C. Clark, ${ }^{5,6,7}$ Bong-Jin Choi $^{1}$

${ }^{1}$ Department of Statistics, North Dakota State University, Fargo, ND, USA; ${ }^{2}$ Department of Health, Nutrition, and Exercise Sciences, North Dakota State University, Fargo, ND, USA;

${ }^{3}$ Department of Education, Health and Behavior Studies, University of North Dakota, Grand Forks, ND, USA; ${ }^{4}$ Alliance for Research in Exercise, Nutrition and Activity, School of Health Sciences, University of South Australia, Adelaide, SA, Australia; ${ }^{5}$ Ohio Musculoskeletal and Neurological Institute, Ohio University, Athens, OH, USA; ${ }^{6}$ Department of Biomedical

Sciences, Ohio University, Athens, OH, USA; ${ }^{7}$ Department of Geriatric Medicine, Ohio University, Athens, OH, USA

\section{Corresponding Author}

Brenda M. Vincent

Department of Statistics

North Dakota State University

1230 Albrecht Blvd

Fargo, ND, USA 58108

Phone: 701-231-7532

Fax: 701-231-8734

Email: brenda.vincent@ndsu.edu 


\begin{abstract}
Background: Engaging in healthy behaviors may help to preserve function during aging; however, it is not well understood how sleeping time is associated with functional capacity in older adults.
\end{abstract}

Aims: We sought to determine the association of sleeping time on functional limitation in a national sample of older Americans.

Methods: The analytical sample included 6,020 adults aged at least 65 years who participated in the 2007-2016 waves of the National Health and Nutrition Examination Survey. Respondents indicated their hours of sleep/week night and were categorized as $<5,5-6.5,7-8,8.5-9$, and $>9$ hours of sleep/week night. Ability to complete 19 functional tasks including basic activities of daily living, instrumental activities of daily living, leisure and social activities, lower extremity mobility activities, and general physical activities were also self-reported. A covariate-adjusted logistic model analyzed the associations between each sleeping time category and functional limitation.

Results: Relative to those reporting 7-8 hours of sleep/week night, older Americans reporting $<5,5-6.5,8.5-9$, and $>9$ hours of sleep/week night had $2.02(95 \%$ confidence interval $(\mathrm{CI}): 1.26$, 3.21), 1.25 (CI: 1.02, 1.53), 1.60 (CI: 1.21, 2.12), and 3.21 (CI: 2.12, 4.87) greater odds for functional limitation, respectively.

Discussion: Sleep should be recognized as a health factor that may reflect functional capacity in older adults. Healthcare providers should discuss the importance of optimal sleep with their older patients and older adults should practice healthy sleeping behaviors for preserving function. 
Conclusions: Not meeting optimal sleep recommendations is associated with functional limitations in older Americans.

Keywords: Aging; Epidemiology; Geriatrics; Health; Life Style; Sleep; Physical Function 


\section{INTRODUCTION}

Functional capacity is often measured by questionnaires where respondents self-report their ability to complete tasks that are generally necessary for autonomous living or basic selfcare [1]. Living with a functional limitation increases the risk for other health concerns such as depression, hospitalization, and early all-cause mortality [2-4]. Moreover, those living with functional limitations have higher medical costs than those without functional limitations [5]. The proportion of adults with functional limitations have increased over time likely due to the increasing proportions of older adults, who generally have higher rates of functional limitations $[6,7]$. Age is a primary risk factor for functional limitations, and the older adult population in the United States is projected to increase by about $112 \%$ by the year $2060[8,9]$. Therefore, lifestyle behaviors that preserve functional capacity should be encouraged during aging.

Restorative sleep has emerged as an important lifestyle behavior for good health [10]. Although older adults benefit from the health characteristics of restorative sleep, they experience age-related changes to their sleep architecture that may influence their sleeping patterns [11]. Sleep problems and poor sleep quality have shown to be associated with functional limitations in

older adults. For example, a prospective cohort study of 3,620 adults aged 24-75 years found that self-reported chronic sleeping problems increased the risk for functional deficits over a 10 -year study period [12]. Likewise, a cross-sectional investigation of 213 adults 65 years and older revealed that self-reported poor sleep quality was associated with functional limitations [13]. These observations are also conceptually consistent with findings highlighting the role of sleep in motor memory consolidation [14-16]. Given that sleeping problems and quality are associated with functional declines, not meeting or exceeding sleep recommendations (i.e., time spent sleeping) may also be linked to diminished functional capacity. 
Promoting healthy lifestyle behaviors such as adequate restorative sleep may help to improve health during aging; however, sleep is not well studied in older adults [17]. Understanding how under- and over-sleeping is associated with not only functional limitations, but also limitations in the individual aspects of function, may help to provide insights into how sleep is linked to functional capacity. Such findings may help to guide intervention strategies for preserving function during aging. Accordingly, the purposes of this study were to determine the associations of sleeping time and 1) functional limitation, and 2) limitations in each aspect of functional in a national sample of older Americans.

\section{METHODS}

\section{Participants}

Publicly available data from 7,013 adults aged at least 65 years who participated in the 2007-2008, 2009-2010, 2011-2012, 2013-2014, or 2015-2016 cross-sectional waves of the National Health and Nutrition Examination Survey (NHANES) were analyzed. The NHANES is a program of studies designed to examine the health and nutritional status of American children, adolescents and adults [18]. Mobile examination centers and survey interviewers traveled to various locations throughout the United States. Health interviews were completed in respondents' homes using computer-aided interview systems, and participants visited mobile examination centers for enhanced exams [19].

To produce reliable data that were representative of all ages and ethnicities in the United States, oversampling occurred in older adults, non-Hispanic Asians, non-Hispanic Blacks and Hispanics [18]. Overall interview response rates for each wave of the NHANES included in our

analyses were above $61 \%$ [20]. A complex, four-stage probability sample design was utilized by 
the NHANES to create a representative sample of non-institutionalized persons residing in the United States. Sample weights were used in our analyses to produce an unbiased national estimate and account for the sampling methods [21, 22]. Each participant provided written informed consent, and NHANES protocols were approved by the National Center for Health Statistics Research Ethnics Review Board.

\section{Response Variables}

Respondents told interviewers if they had no difficulty, some difficulty, much difficulty, or were unable to complete 19 tasks from five different aspects of function. These aspects included: basic activities of daily living (BADL; "getting in and out of bed", "using a fork, knife, and cup", "walking between rooms on the same floor", “dressing yourself”), instrumental activities of daily living (IADL; "house chores", "managing money", "preparing meals"), leisure and social activities ("going out to movies and events", "leisure activities at home", "attending social events"), lower extremity mobility activities ("walking up 10 steps", "walking for a quarter mile"), and general physical activities ("grasping or holding small objects", "lifting or carrying", "reaching up and over the head", "sitting for long periods of time", "standing for long periods of time", "standing up from an armless chair", "stooping, crouching, kneeling").

Those reporting that they had some difficulty, much difficulty, or were unable to perform any of the 19 tasks were considered as having a functional limitation. Similarly, respondents that indicated some difficulty, much difficulty, or were unable to perform any task within each of the five aspects of function were considered as having a limitation in that individual aspect. Several studies have used such criteria for defining functional limitations with NHANES data [23-27]. Those without information for functional limitations were excluded $(n=14)$. 


\section{Explanatory Variables}

Interviewers asked respondents "How much sleep do you usually get at night on weekdays or workdays". Open ended responses to the nearest 30 minutes were used to categorize participants' sleeping time based on national sleep recommendations for older adults [28]. The "recommended" amount of sleep according to the National Sleep Foundation guidelines for older adults are 7-8 hours [28]. It is "not recommended" for older adults to get $<5$ or $>9$ hours of sleep and "may be appropriate" for older adults to get between 5-6 or 9 hours of sleep [28]. Therefore, using these recommendations, we created the following categories: $<5,5-6.5,7-8,8.5-9$, and $>9$ hours of sleep. Those without information for sleep were excluded $(n=22)$.

\section{Covariates}

Age, sex, race and ethnicity (Hispanic, non-Hispanic Black, non-Hispanic White, other) and marital status (married, not married). Standing height was measured with a fixed stadiometer and body mass was collected with a digital scale (Mettler-Toledo International Inc.; Columbus, OH, USA). Body mass index was calculated as body mass in kilograms divided by height in meters-squared $\left(\mathrm{kg} / \mathrm{m}^{2}\right)$. Those with a body mass index of $\geq 30 \mathrm{~kg} / \mathrm{m}^{2}$ were considered obese. Respondents told interviewers if they had smoked at least 100 cigarettes in their lifetime (smoking history) and/or if they were currently cigarette smokers. Respondents also indicated if a doctor had ever diagnosed them with diabetes. A single-item measure of self-rated health was used and respondents rated their general health as "excellent", "very good", "good", "fair", or "poor".

The well-validated 9 item Patient Health Questionnaire was used to identify depressive symptoms $[29,30]$. Participants reported how often (0: not at all; 1: several days; 2 : more than 
half the days; 3 : nearly every day) they were bothered by the following in the previous two weeks: "having little interest or pleasure in doing things", "feeling down, depressed or hopeless", "trouble with sleeping or sleeping too much", "feeling tired or having little energy", "poor appetite or over eating", "feeling bad about yourself", "trouble concentrating on things", "moving or speaking slowly or too fast", and "thoughts that you would be better off dead or of hurting yourself'. Scores ranged from $0-27$ and those with scores $\geq 10$ were considered depressed [29]. Those without information for one or more covariates were excluded ( $\mathrm{n}=957)$.

\section{Statistical Analyses}

All analyses were conducted with SAS 9.4 software (SAS Institute; Cary, NC). A logistic regression model that accounted for the sample weights (PROC SURVEYLOGISTIC) determined the associations between each of the sleep categories with functional limitations. The sample weights were included in the PROC SURVEYLOGISTIC statement using the "WEIGHT" option. To maintain the integrity of the NHANES sampling design, and thus the survey weights, the "DOMAIN" option was used to perform analyses only on eligible participants. Separate logit models (PROC SURVEYLOGISTIC) also analyzed the associations between sleeping time categories and limitations in the individual aspects of function (BADL;

IADL; leisure and social activities; lower extremity mobility activities; general physical activities). Those categized as meeting national sleep recommendations for older adults (7-8 hours) [28] were the reference group and each of the logistic models were adjusted for age, sex, race and ethnicity, obesity, smoking status, diabetes diagnosis, self-rated health, and depression status. An alpha level of 0.05 was used for all analyses.

\section{RESULTS}


There were 6,020 participants across the five NHANES waves analyzed (1,319 in the 2007-2008 wave; 1,270 in the 2009-2010 wave; 1,058 in the 2011-2012 wave; 1,162 in the 20132014 wave; 1,211 in the 2015-2016 wave). This corresponded to a weighted total of 180,793,089 (31,138,452 in the $2007-2008$ wave; $33,287,082$ in the $2009-2010$ wave; $34,301,029$ in the 2011 2012 wave; $39,022,884$ in the $2013-2014$ wave; $43,043,642$ in the $2015-2016$ wave). The descriptive characteristics of the 6,020 participants are shown in Table 1 . Of those participants, $276(4.6 \%)$ had $<5$ hours/sleep week night, 1,538 (25.5\%) had 5-6.5 hours of sleep/week night, 3,121 (51.8\%) had 7-8 hours of sleep/week night, 647 (10.8\%) had 8.5-9 hours of sleep/week night, and $438(7.3 \%)$ had $>9$ hours of sleep/week night. To make comparisons between those in each sleeping time category for the descriptive characteristics, the means and $95 \%$ confidence intervals (CI) are shown in Appendix 1. Those reporting 7-8 hours of sleep/week night had a significantly lower proportion of functional limitation (67.5\%; CI: 65.9\%, 69.2\%) compared to older Americans in the $<5$ (84.7\%; CI: 80.5\%, 89.0\%), 5-6.5 (73.1\%; CI: 70.9\%, 75.3\%), 8.5-9 (76.5\%; CI: 73.2\%, 79.7\%), and >9 (88.1\%; CI: 85.1\%, 91.1\%) hours of sleep/week night categories. Figure 1 presents the proportions of participants with functional limitation by hours of nightly sleep.

Figure 2 depicts the results for the association between the sleeping time categories and functional limitation. Compared to those engaging in 7-8 hours of sleep/week night, older Americans with $<5,5-6.5,8.5-9$, and $>9$ hours of sleep/week night had 2.02 (CI: 1.26, 3.21), 1.25 (CI: 1.02, 1.53), 1.60 (CI: 1.21, 2.12), and 3.21 (CI: 2.12, 4.87) greater odds for functional limitation, respectively.

Table 2 presents the results for the association between the sleeping time categories and limitations in each aspect of functional. Those engaging in $<5$ hours of sleep/week night had 
increased odds for limitations in each aspect of functional: 1.50 (CI: 1.03, 2.20) for instrumental activities of daily living, 1.54 (CI: 1.02, 2.32) for general physical activities, 1.61 (CI: 1.07, 2.42) for activities of daily living, 1.94 (CI: 1.28, 2.92) for leisure and social activities, and 2.14 (CI: $1.43,3.21)$ for lower extremity mobility activities. Similarly, older Americans that had 8.5-9 hours of sleep/week night had increased odds for limitations in each aspect of functional: 1.31 (CI: 1.01, 1.68) for activities of daily living, 1.32 (CI: 1.03, 1.69) for lower extremity mobility activities, 1.51 (CI: 1.15, 1.98) for general physical activities, 1.51 (CI: 1.16, 1.95) for leisure and social activities, and 1.69 (CI: 1.32, 2.17) for instrumental activities of daily living. Moreover, those indicating $>9$ hours of sleep/week night had increased odds for limitations in each aspect of functional: 2.36 (CI: 1.74, 3.21) for lower extremity mobility activities, 2.49 (CI: $1.74,3.55)$ for general physical activities, 2.62 (CI: 1.95, 3.54) for activities of daily living, 2.92 (CI: 2.20, 3.87) for instrumental activities of daily living, and 3.29 (CI: $2.48,4.38$ ) for leisure and social activities. However, there were no significant associations for those engaging in 5-6.5 hours of sleep/week night and limitations in each aspect of functional.

\section{DISCUSSION}

The principal results of this investigation revealed that sleeping time was associated with functional limitation in a national sample of older Americans. Specifically, those engaging in $<5$, 5-6.5, 8.5-9, and $>9$ hours of sleep/week night had $102 \%, 25 \%, 60 \%$, and $221 \%$ increased odds for functional limitation compared to those that had 7-8 hours of sleep/week night, thereby representing a "U-shaped" sleeping time trend. A similar trend was observed when examining the associations between sleeping time and limitations in each aspect of functional, such that those who engaged in $<5,8.5-9$, or $>9$ hours of sleep/week night had increased odds for limitations in each aspect of functional. We suggest that health care providers discuss the 
importance of proper sleeping time for preserving function with their older adult patients. Older adults should also incorporate healthy sleeping habits in their lifestyles for helping to preserve functional capacity.

Our findings align with other previous investigations wherein chronic sleeping problems and sleep quality were associated with functional limitations [12, 13]. Chronic sleeping problems and sleep quality may influence sleeping time [31], which has also been shown to be associated with functional deficits in older adults [32]. A different cross-sectional investigation revealed that middle-aged Americans with a functional limitation had an elevated prevalence of sleep disorders compared to those without a functional limitation [33]. These findings may suggest that excessive sleep could be a consequence of functional declines in older populations. Given that living with a functional limitation may lead to sleeping problems, and that our cross-sectional analyses suggest under and over sleeping is associated with functional limitations, it is challenging to determine how exactly sleeping time and functional limitations are associated. Future research should examine the longitudinal and directional associations of sleep and functional limitations, and if there are any other variables that may help to explain such associations.

Approximately $26 \%$ of older Americans get less than seven hours of sleep each day [34]. Insomnia and related sleeping problems are influential for quality of life during aging, which may also factor into social functioning. For example, a 3-year longitudinal study of 10,430 women aged 70-75 years determined that problems with sleeping were associated with poorer social functioning [35]. Neuroticism and other similar personality characteristics have also been shown to be predictive of poor sleep [36]. This may help to explain why our findings showed that those in certain sleeping time categories had increased odds for a limitation in leisure and 
social activities. While under and over sleeping may influence an older adult's ability to complete leisure and social activities, sleeping time may also have implications on other aspects of functional capacity.

Short and prolonged periods of sleeping time are associated with fatigue and lack of physiological stressors such as physical activity $[37,38]$. This may help to explain why our findings demonstrated that those who could be considered under or over sleepers had greater odds for limitations in more physically-driven tasks such as BADL, lower extremity mobility activities, and general physical activities. Short and excessive sleep time is also associated with lower cognitive functioning [39, 40], which may explain why our findings indicated sleeping time was associated with limitations in neuropsychologically-driven tasks such as IADL. Moreover, poor environmental and interpersonal factors are linked to sleep [41], which may explain why our results suggest that those in certain sleeping time categories were associated with limitations in leisure and social activities, even after adjusting for depression.

Our findings are also consistent with results that highlight the role of sleep in motor memory consolidation $[14,15]$. While memory consolidation may occur while awake [42], the greatest portion of consolidation may occur preferentially or exclusively during sleep. For example, sleep has been shown to enhance motor sequence learning in young adults, but this effect is reportedly reduced in older adults [14]. This finding, when coupled with others [14, 15], suggests that age-related changes in sleep or networks activated during encoding or during sleep contribute to declines in motor sequence consolidation [16].

Indeed, our findings suggest that older Americans that engaged in $<5,5-6.5,8.5-9$, and $>9$ hours of sleep/week night had increased odds for functional limitation relative to those with 7-8 hours of sleep/week night. A similar trend was observed when evaluating the sleeping time 
categories and each aspect of functional limitation, such that those who engaged in $<5,8.5-9$, and $>9$ hours of sleep/week night had increased odds for limitations in each aspect of function. Our results align with those indicating a "U-shaped" curve exists for the association between sleep duration and health [43], and support public health programs aiming to highlight the health benefits of sleep [44]. We suggest that healthcare providers communicate the importance of optimal sleep for preserving function with their older patients. Likewise, older adults should practice optimal sleep as a lifestyle behavior to help preserve function.

Some limitations should be acknowledged. The NHANES utilized a cross-sectional design, and thus, we were unable to make longitudinal or cause-effect inferences. Although selfreport data are common in large population-based studies such as the NHANES, self-report information is subject to bias. The 9 item Patient Health Questionnaire, a common measure of depressive symptoms for NHANES studies, was also used in our investigation as a covariate. However, the Patient Health Questionnaire item that inquires about "trouble with sleeping or sleeping too much" may have overlapped with our explanatory variable. While other measures for assessing functional limitations may exist, the NHANES utilizes the 19-item functional limitation questionnaire. The NHANES only collects sleep data for weeknights, which may not capture sleeping time during naps or weekend nights. However, total sleeping time and time spend in bed generally does not change between weekdays and weekend days in older populations [45].

\section{Conclusions}

The results of our investigation indicate that older Americans under and over the sleep recommendations have greater odds of functional limitation. Similar findings existed when evaluating the sleeping time categories and limitations in individual aspects of function. Sleep 
should be recognized as a factor that influences function and that a "U-shaped" sleeping time trend appears to exist for the association between sleeping time and functional capacity. We suggest that healthcare providers discuss the importance of sleep for health and functioning with their older patients. Older adults should incorporate healthy sleeping behaviors in their lifestyles for preserving function. Doing so may help to decelerate the disabling process in the rapidly growing aging American population.

\section{Compliance with Ethical Standards}

Conflict of Interest: The authors have no funding disclosures or conflicts of interest to report.

Statement of Human and Animal Rights: NHANES protocols were approved by the National

Center for Health Statistics Research Ethnics Review Board. Approved protocol numbers can be found on the NHANES website [46].

Informed Consent: Written informed consent was provided by participants before entering the NHANES.

Ethical Approval: NHANES data analyzed in this study are publicly available and protocols used to collect these data have been approved by the National Center for Health Statistics Research Ethnics Review Board.

Funding: None.

Acknowledgements: None.

\section{REFERENCES}

1. Mlinac ME, Feng MC. Assessment of activities of daily living, self-care, and independence. Arch Clin Neuropsychol. 2016;31(6):506-16. 
2. He M, Ma J, Ren Z, et al. Association between activities of daily living disability and depression symptoms of middle-aged and older Chinese adults and their spouses: A community based study. J Affect Disord. 2019;242:135-42.

3. McGrath RP, Vincent BM, Lee I-M, Kraemer WJ, Peterson MD. Handgrip Strength, Function, and Mortality in Older Adults: A Time-Varying Approach. Med Sci Sports Exerc. 2018;50(11):2259-66.

4. Covinsky KE, Pierluissi E, Johnston CB. Hospitalization-associated disability:"She was probably able to ambulate, but I'm not sure”. JAMA. 2011;306(16):1782-93.

5. Peterson MD, Mahmoudi E. Healthcare utilization associated with obesity and physical disabilities. American journal of preventive medicine. Am J Prev Med. 2015;48(4):42635.

6. Chen Y, Sloan FA. Explaining Disability Trends in the US Elderly and Near-Elderly Population. Health Serv Res. 2015;50(5):1528-49.

7. Stevens AC. Adults with one or more functional disabilities-United States, 2011-2014. MMWR Morb Mortal Wkly Rep. 2016;65.

8. Colby SL, Ortman JM. Projections of the Size and Composition of the US Population: 2014 to 2060. Current Population Reports. 2014;P25-1143.

9. Connolly D, Garvey J, McKee G. Factors associated with ADL/IADL disability in community dwelling older adults in the Irish longitudinal study on ageing (TILDA). Disabil Rehabil. 2017;39(8):809-16.

10. Buysse DJ. Sleep health: can we define it? Does it matter? Sleep. 2014;37(1):9-17.

11. Miner B, Kryger MH. Sleep in the aging population. Sleep Med Clin. 2017;12(1):31-8. 
12. Friedman EM. Self-reported sleep problems prospectively increase risk of disability: Findings from the survey of Midlife Development in the United States. J Am Geriatr Soc. 2016;64(11):2235-41.

13. Chien M-Y, Chen H-C. Poor sleep quality is independently associated with physical disability in older adults. J Clin Sleep Med. 2015;11(03):225-32.

14. Bottary R, Sonni A, Wright D, Spencer RM. Insufficient chunk concatenation may underlie changes in sleep-dependent consolidation of motor sequence learning in older adults. Learn Mem. 2016;23(9):455-9.

15. Pace-Schott EF, Spencer RM. Age-related changes in consolidation of perceptual and muscle-based learning of motor skills. Front Aging Neurosci. 2013;5:83.

16. Wilson JK, Baran B, Pace-Schott EF, Ivry RB, Spencer RM. Sleep modulates word-pair learning but not motor sequence learning in healthy older adults. Neurobiol Aging. 2012;33(5):991-1000.

17. Dzierzewski JM, Dautovich ND. Who Cares about Sleep in Older Adults? Clin Gerontol. 2018;41(2):109-12.

18. National Health and Nutrition Examination Survey. Overview. https://www.cdc.gov/nchs/data/nhanes/nhanes_13_14/NHANES_Overview_Brochure.pd f. Accessed December 3, 2019.

19. National Health and Nutrition Examination Survey: Plan and Operations, 1999-2010. https://www.cdc.gov/nchs/data/series/sr_01/sr01_056.pdf. Accessed December 3, 2019.

20. Centers for Disease Control and Prevention. Response Rates. https://wwwn.cdc.gov/nchs/nhanes/ResponseRates.aspx\#response-rates. Accessed December 3, 2019. 
21. National Health and Nutrition Examination Survey: Analytic Guidelines, 2011-2014 and 2015-2016. https://wwwn.cdc.gov/nchs/data/nhanes/20112012/analyticguidelines/analytic_guidelines_11_16.pdf. Accessed December 3, 2019.

22. National Health and Nutrition Examination Survey: Analytic Guidelines, 1999-2010. https://www.cdc.gov/nchs/data/series/sr_02/sr02_161.pdf. Accessed December 3, 2019.

23. Hajjar I, Wharton W, Mack WJ, Levey AI, Goldstein FC. Racial disparity in cognitive and functional disability in hypertension and all-cause mortality. Am J Hypertens. 2015;29(2):185-93.

24. Jindai K, Nielson CM, Vorderstrasse BA, Quiñones AR. Multimorbidity and Functional Limitations Among Adults 65 or Older, NHANES 2005-2012. Prev Chronic Dis. 2016;13:E151.

25. Wu L-W, Chen W-L, Peng T-C, et al. All-cause mortality risk in elderly individuals with disabilities: a retrospective observational study. BMJ Open. 2016;6(9):e011164.

26. Krok-Schoen J, Price AA, Luo M, Kelly O, Taylor CA. Low dietary protein intakes and associated dietary patterns and functional limitations in an aging population: A NHANES analysis. J Nutr Health Aging. 2019;23(4):338-47.

27. McGrath R, Stastny S, Casperson S, Jahns L, Roemmich J, Hackney KJ. Daily Protein Intake and Distribution of Daily Protein Consumed Decreases Odds for Functional Disability in Older Americans. J Aging Health. 2019:0898264319881864.

28. National Sleep Foundation. National Sleep Foundation Recommends New Sleep Times. https://www.sleepfoundation.org/press-release/national-sleep-foundation-recommendsnew-sleep-times. Accessed December 3, 2019. 
29. Kroenke K, Spitzer RL, Williams JB. The PHQ-9: validity of a brief depression severity measure. J Gen Intern Med. 2001;16(9):606-13.

30. Kroenke K, Spitzer RL. The PHQ-9: a new depression diagnostic and severity measure. Psychosom Med. 2002;64(2):258-66.

31. Buysse DJ. Insomnia. JAMA. 2013;309(7):706-16.

32. Lorenz RA, Budhathoki CB, Kalra GK, Richards KC. The relationship between sleep and physical function in community-dwelling adults: a pilot study. Fam Community Health. 2014;37(4):298-306.

33. Whitney DG, Hurvitz EA, Peterson MD. Cardiometabolic disease, depressive symptoms, and sleep disorders in middle-aged adults with functional disabilities: NHANES 20072014. Disabil Rehabil. 2018:1-6.

34. Centers for Disease Control and Prevention. Sleep and Sleep Disoreders. https://www.cdc.gov/sleep/data_statistics.html. Accessed December 3, 2019.

35. Byles JE, Mishra GD, Harris MA, Nair K. The problems of sleep for older women: changes in health outcomes. Age Ageing. 2003;32(2):154-63.

36. Duggan KA, Friedman HS, McDevitt EA, Mednick SC. Personality and healthy sleep: the importance of conscientiousness and neuroticism. PLoS One. 2014;9(3):e90628.

37. Grandner MA, Drummond SP. Who are the long sleepers? Towards an understanding of the mortality relationship. Sleep Med Rev. 2007;11(5):341-60.

38. Kline CE. The bidirectional relationship between exercise and sleep: implications for exercise adherence and sleep improvement. Am J Lifestyle Med. 2014;8(6):375-9.

39. Wild CJ, Nichols ES, Battista ME, Stojanoski B, Owen AM. Dissociable effects of selfreported daily sleep duration on high-level cognitive abilities. Sleep. 2018;41(12). 
40. Alhola P, Polo-Kantola P. Sleep deprivation: Impact on cognitive performance. Neuropsychiatr Dis Treat. 2007;3(5):553-67.

41. Grandner MA. Sleep, health, and society. Sleep Med Clin. 2017;12(1):1-22.

42. Diekelmann S, Born J. The memory function of sleep. Nat Rev Neurosci. 2010;11(2):114-26.

43. Cappuccio FP, D'Elia L, Strazzullo P, Miller MA. Sleep duration and all-cause mortality: a systematic review and meta-analysis of prospective studies. Sleep. 2010;33(5):585-92.

44. Healthy People 2020. Sleep Health. https://www.healthypeople.gov/2020/topicsobjectives/topic/sleep-health. Accessed December 3, 2019.

45. Hashizaki M, Nakajima H, Kume K. Monitoring of weekly sleep pattern variations at home with a contactless biomotion sensor. Sensors (Basel). 2015;15(8):18950-64.

46. Centers for Disease Control and Prevention. NCHS Research Ethics Review Board (ERB) Approval. https://www.cdc.gov/nchs/nhanes/irba98.htm. Accessed December 3, 2019. 


\section{FIGURE LEGEND}

Figure 1. Proportions of Participants with Functional Limitation by Hours of Nightly Sleep.

Note: Hours of nightly sleep is rounded down to the nearest whole number.

Figure 2. Associations Between Sleeping Time Categories and Functional Limitation.

Note: The model was adjusted for age, sex, race and ethnicity, obesity, smoking status, diabetes, self-rated health, and depression status. 